\title{
Spectroscopy of biological nanocrystals
}

\author{
Inanc Ortac ${ }^{\mathrm{a}}$ and Feride Severcan ${ }^{\mathrm{b}, *}$ \\ ${ }^{a}$ Department of Physics, Middle East Technical University, 06531 Ankara, Turkey \\ ${ }^{\mathrm{b}}$ Department of Biology, Middle East Technical University, 06531 Ankara, Turkey
}

\begin{abstract}
Nanocrystals have gained much interest in recent years, due to their unusual properties allowing interesting applications in physical and biological science. In this literature review, biological nanocrystals are discussed from the spectroscopic point of view. Firstly, the theory behind the outstanding abilities of the nanocrystals is described. Secondly, the spectroscopic properties of biological nanocrystals are mentioned. Lastly, the use of nanocrystals with various spectroscopic applications is reviewed such as biosensor applications with UV-visible and surface plasmon resonance and Raman spectroscopy of biological materials. Finally, a short discussion of infrared nanocrystals and their abilities are included in the review.
\end{abstract}

Keywords: Quantum dots, nanoparticles, spectroscopy

\section{Introduction}

World science has already entered in the nanoscience era. Many branches of science have met the opportunities that nanoscale provided. Growing nanoscale science has allowed to build up or to alter materials starting from the smallest pieces. New building blocks and tools are being developed. Today, one of the most important nano structures are the nanocrystals (NCs) which have found many areas of application in physics, material science and biology. NCs have found area of utilization as probes for visualization of molecules, as tools for enhancing signals in spectroscopic applications, or as building blocks for complex nano and micro structures.

NCs are tiny crystal structures, generally manufactured from metals, semiconductors or insulators [1]. They are consisted of a few hundreds of atoms forming a crystal structure and several electrons are trapped inside this structure. NCs can be made up of various materials such as insulators, metals and semiconductors. Semiconductor NCs, or generally referred as quantum dots (QDs), can be formed inside a medium (Fig. 1A) or by colloidal synthesis (Fig. 1B) as well as the metal nanoparticles. Colloidal NCs such as colloidal QDs or metal nanoparticles are not bound to any larger crystal; and mostly they are in the form of spheres. Their radius varies from a few to several hundreds of nanometers. The QDs inside a medium are currently used for physical applications namely, for photonic sensors, electronic applications or QD lasers and LEDs, etc. [2-6]. On the other hand, colloidal NCs recently have been utilized in important applications in biology and medicine, particularly for biological labeling, biosensor [7-13] or nanotechnological applications (nanocrystals conjugated with biomolecules) [14-21]. Currently, there is a wide research worldwide for physical applications of nanocrystal. However, there has been no significant commercial product so far. On the contrary, bioscience and medicine have utilized the nanocrystals in numerous applications. NCs have been used for several years in research [1,9,22-25] and different types of QDs to be used for biological labeling applications can be purchased from several suppliers [4].

\footnotetext{
*Corresponding author. Tel.: +90 31221051 66; Fax: +90 312210 79 76; E-mail: feride@ @etu.edu.tr.
} 


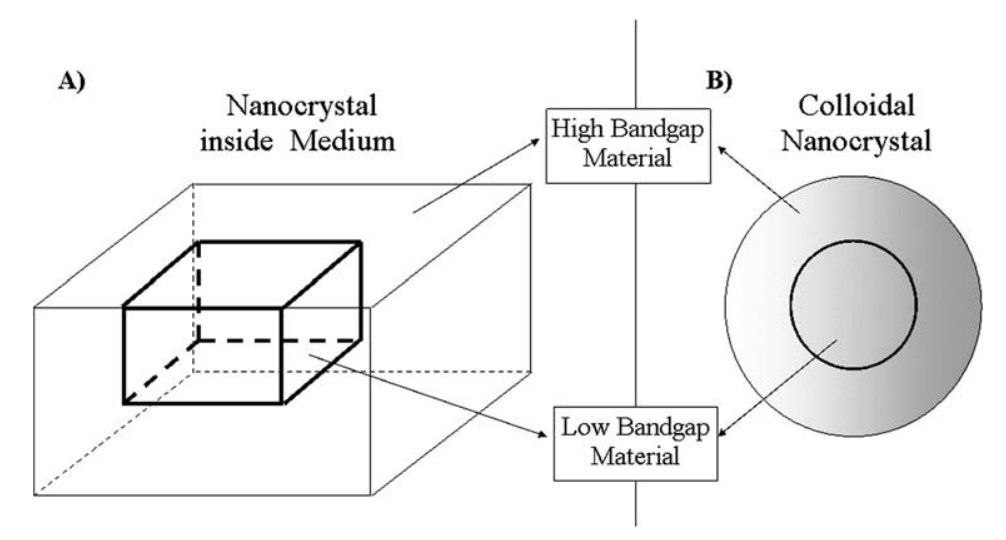

Fig. 1. (A) An illustration of a nanocrystal formed inside a larger medium. The shape of the nanocrystal varies depending on the fabrication method. The medium is generally crystal. However, it is possible to form nanocrystal inside amorphous structures. (B) Free standing nanocrystal, which is mostly used in biological applications. Although the outer layer is not necessary, this structure increases the encapsulation efficiency.

\section{The theory behind the properties of nanocrystals}

The unusual properties of nanocrystals depend on two basics: Encapsulation of electrons in a dimension in the order of its De Broglie wavelength, defining the wave nature of the electrons, and the effective mass of the electron in a crystal structure. In natural atoms, electrons are trapped by a central potential. If an electron is encapsulated in a dimension which is comparable to its De Broglie wavelength, then the size quantization occurs which makes the electron's energy levels become discrete in that dimension [26]. If this encapsulation occurs in three dimensions, the energy levels of the electron are totally quantized. By the central potential of the natural atoms the electrons are confined in small dimensions resulting in the energy levels that we observe. In free space, the De Broglie wavelength is an extremely small scale to capture electron artificially. However, the concept of effective mass of electrons in crystals overcomes this problem. De Broglie wavelength of a particle inside a crystal medium depends on the mass of the particle which can be expressed as, $\lambda=h / \sqrt{\left(3 m_{\text {eff }} k T\right)}$ [27]. Therefore, the electrons in a crystal have a different mass, which is much smaller than the mass of the electron in free space. This is called the effective mass and results mainly from the electrostatic interaction of the lattice atoms. Effective mass of the electron varies from crystal to crystal. If GaAs is considered, the effective mass of the electron could fall below 7\% of the free electron mass [28]. Accordingly, if an electron is captured within a dimension of 100 angstroms, it is possible to observe discrete energy levels of the electron resembling the ones in natural atoms [28]. This is like deceiving electrons or emulation of the natural atoms in crystals. In NCs, the encapsulation of electrons can be provided by creating tiny nanostructures of crystal that is covered with another outer crystal having higher conduction band energy or higher bandgap (energy difference between conduction band and valence band) than the inner one. Then, the electrons will prefer to be in the crystal having lower conduction band energy, or simply by creating free or bound nanocrystals in a dielectric medium like air can provide encapsulation of electrons [29]. The motivation of developing NCs depends on controllable size, shape and the number of electrons inside. Thus, by the development of the NC technology it would be possible to make structures that their emission and absorption wavelengths with their sizes and shapes are controllable to be used in different applications. These properties of NCs have allowed tremendous number of applications and uses to 
emerge in various practices. The biological applications of NCs will be discussed in the following parts of this letter.

\section{Biological nanocrystals}

Applications of nanocrystals in biology can be classified mainly in two topics. One is using nanocrystals as probes for biological labeling applications and for increasing the spectroscopic signal for spectroscopy or microscopy $[1,22,30]$, the other is the grouping of nanocrystals using biological molecules as templates [31] for electronic and photonic applications [14,15,17,32,33] or for building nanostructures $[16,18,19,32,34]$.

The outstanding spectroscopic and microscopic properties of QDs, which will be mentioned later in the text, have resulted in many practices of QDs in biological labeling and provided preference of QDs over conventional organic probes. Moreover, many studies have shown that combining nanocrystals, mainly metal nanoparticles, with various biological molecules increases the signal efficiency in different spectroscopic applications. The other main use of nanocrystals is in fact not an application in biology; rather, biological molecules are used to sort, assemble and group QDs or metal nanoparticles in nanotechnological applications.

As nanocrystals are hydrophobic and toxic to the biological systems they must be made biocompatible to be used in mostly aqueous biological environment. To overcome the incompatibility of the surface of the nanocrystals to the biological environment, their surface should be covered with biocompatible molecules. There are various methods to create biocompatible nanocrystals some of which can be listed as encapsulation of dots in chaperonin proteins [7], phospholipids micelles [35], or using bi-functional proteins whose one side is hydrophobic like thiols $(-\mathrm{SH})$ and the other side is hydrophilic like carboxyl $(-\mathrm{COOH})[1]$.

Making nanocrystals biocompatible is not the whole problem. These nanocrystals have to be integrated with other molecules to be used in biological applications. In fact, they should be specific to some substances. Several techniques are being used for conjugation of nanocrystals with biomolecules. These conjugating methods resemble key-lock model. In this model, biomolecules such as antibodies [36], oligonucleotides [18,19] or streptavidin-biotin complexes [37] are used. The use of key-lock model structures depends on the specific binding ability of the molecules in consideration. For instance, an NC covered with an oligonucleotide (i.e. DNA or RNA) having specific base order will only bind to a place including the oligonucleotide having the complementary base sequence [1].

\subsection{Spectroscopic properties of biological nanocrystals}

Since QDs have discrete energy levels resembling natural atoms, they have a wide excitation but a narrow emission spectrum. For instance, CdSe QD shows obviously higher fluorescence intensity than the Latex fluorescent sphere in addition to having a narrower bandwidth of emission peak (Fig. 2A) [38]. This provides preference of quantum dots over organic fluorescence or spectroscopic probes. In spectroscopic studies, the NCs show sharp and high peaks compared to the conventional organic dyes and also shows incomparable success in single molecule studies [22] (Fig. 3). Mostly the spectra overlap while studying with the organic fluorophores. This prevents studying the related activity of several structures. However, the narrow emission bandwidth of NCs allows observation of several molecules at the same time. Several properties of various QDs are depicted in Fig. 4 [39]. 

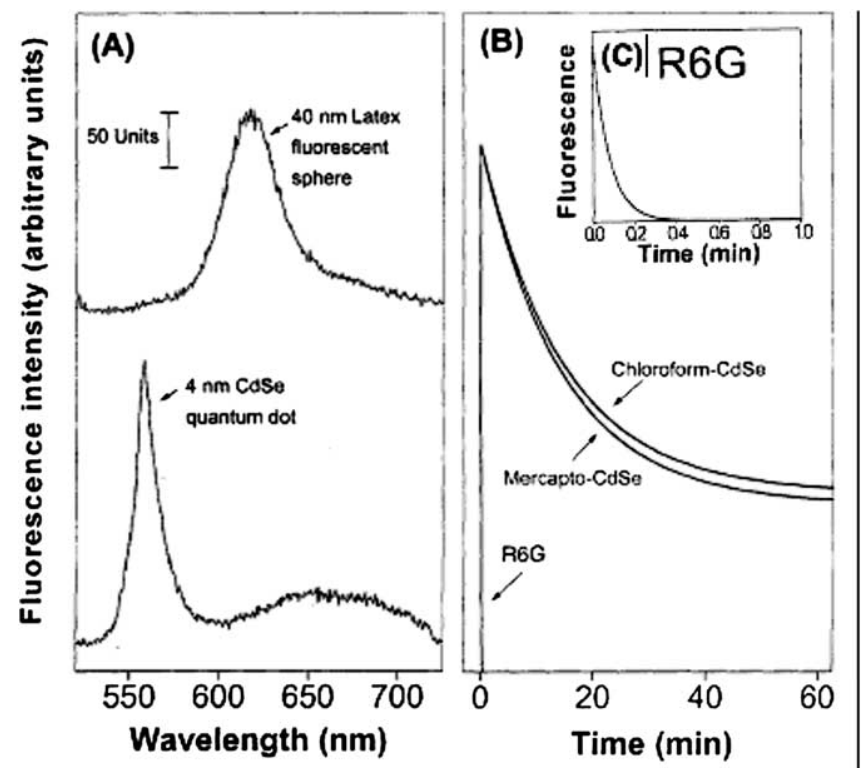

Fig. 2. The representation of the advantage of using luminescent QDs instead of organic dyes in terms of photophysical properties. (A) Wavelength-resolved spectra obtained from a single $40 \mathrm{~nm}$ fluorescent latex and a single mercapto-QD. The broad emission peak at $660 \mathrm{~nm}$ is believed to result from surface defects on the QD. (B) Time-resolved photobleaching curves for the original QDs, the solubilized QDs, and the dye R6G. (C) Detailed curve of the time-resolved photobleaching curve for R6G. Reproduced with permission from Science 281 (1998), 2016-2018.

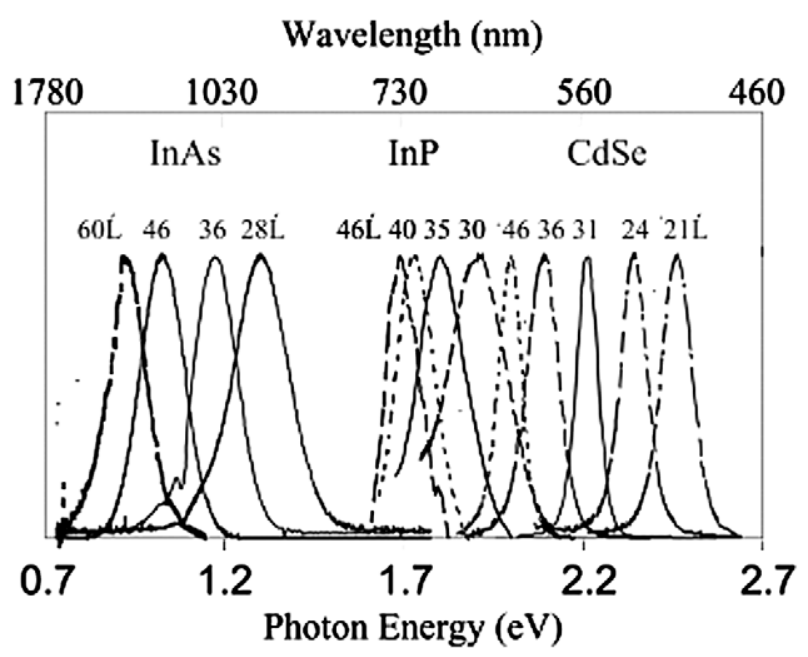

Fig. 3. Representation of the size and composition dependence of the colloidal QDs. Reproduced with permission from Pure Appl. Chem. 72 (2000), 3-9.

The fluorescence wavelength can be changed just by changing the size of the dot [1] (Fig. 3) and unlike conventional fluorescence labels, several fluorescent NCs can be excited by a single excitation source [12]. As a result, the excitation source has a minimal effect to the biological environment while using NC labels. In addition to all, they suffer less from photobleaching [35]. Most of the organic dyes lose their fluorescence intensity completely in minutes (Fig. 5) [35]. This limits the observation of the biological 

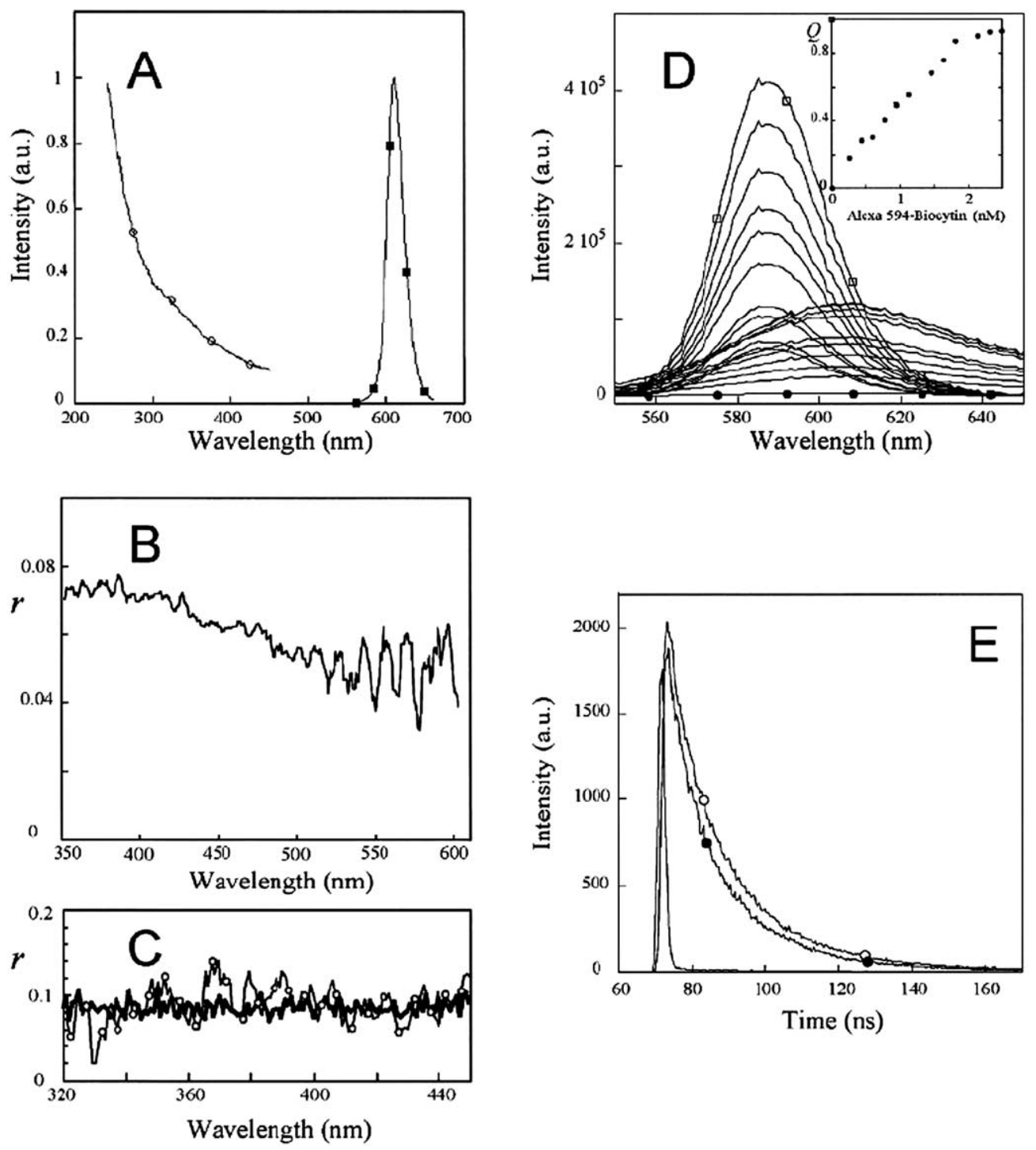

Fig. 4. Spectroscopic properties of QDs. (A) Excitation (circles) and emission (squares) spectra of QD605. (B) Steady-state anisotropy excitation spectrum of QD605. (C) Steady-state anisotropy excitation spectrum in glycerol (circles) and water (thick line). (D) FRET titration. Evolution of the steady-state emission spectra of $2 \mathrm{nM}$ QD585 with increasing concentrations of Alexa594-biocytin after spectral decomposition and substraction of the contribution of the direct excitation of the acceptor. Initial spectrum of QD (squares); sensitized emission of Alexa594 (circles). Inset: Fraction of QD fluorescence quenched $(Q)$ during titration. (E) Time-resolved fluorescence decay of the emission of $2 \mathrm{nM}$ QD585, alone (open circles) and in the presence of 1/2 equivalent of Alexa594-biocytin (closed circles). Reproduced with permission from Mic. Rec. Tech. 65 (2004), 169-179.

phenomena occurring in longer time periods. On the contrary, nanocrystals remain stable and can keep their fluorescence intensity almost constant for longer periods in the scale of hours. Figures $2 \mathrm{~B}$ and $2 \mathrm{C}$ show the comparison of the photobleaching curves of the original QDs, the solubilized QDs, and the dye R6G [38]. Furthermore, Fig. 5 demonstrates the obvious difference of QDs with Green Fluorescent Protein (GFP) [35]. Therefore, they can be used in long time observation or real time in vivo applications $[30,35,40]$. 

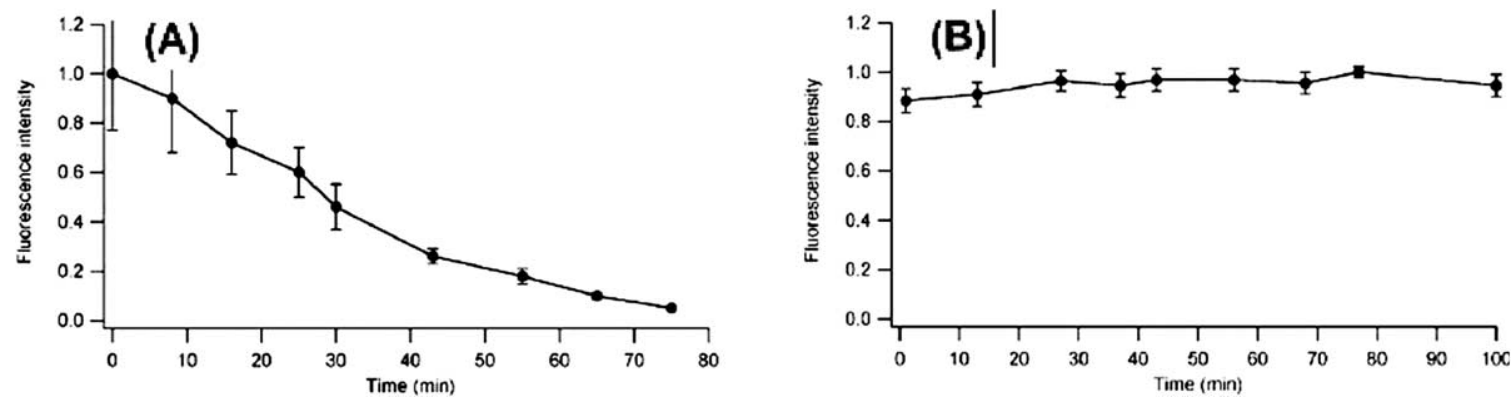

Fig. 5. (A) Fluorescence intensity of GFP. (B) Fluorescence intensity of QD. Both fluorophores are under continuous excitation. $($ Bar $=30 \mu \mathrm{m})$ Reproduced with permission from Science 298 (2002), 1759-1762.

\subsection{Spectroscopic applications of biological nanocrystals}

The encapsulation of nanocrystals inside chaperonin proteins creates the possibility to make biosensor applications. The chaperonin proteins can encapsulate $\mathrm{CdS}$ nanocrystals as well as denatured proteins in a cylindrical cavity. This structure is so stable in the biological environment. Addition of ATP into the environment in the presence of $\mathrm{Mg}^{+}$and $\mathrm{K}^{+}$, causes chaperonin to change its conformation and release the nanocrystal at the end (Fig. 6). This situation can be monitored by size-exclusion chromatography (SEC) with a UV/Fluorescence dual detector (Fig. 7) [7]. In analytical SEC trace there is a UV peak as well as a fluorescence peak for complex chaperonin protein, T.th cpn with CdS. However, after the addition of ATP and $\mathrm{MgCl}_{2}$ the fluorescence peak disappears. The UV peak remains; however there is another UV peak indicating ATP and its hydrolysed products [7].

Using metallic NCs combined with UV-visible and surface plasmon resonance (SPR) spectroscopy is a fruitful combination for chemical biosensor applications [37,41] and for observing protein conformational changes [13].

By using localized SPR (LSPR) spectroscopy with biotinylated surface-confined Ag nano-triangles, a sensitive detection system for streptavidin can be established and anti-biotin binding to biotinylated $\mathrm{Ag}$ nano-triangles can be monitored. There are clear peak shifts in the LSPR spectra when Ag nanoparticles are modified by $1 \mathrm{mM} \mathrm{1:3} \mathrm{11-MUA} \mathrm{(11-mercaptoundecanoic} \mathrm{acid):1-OT} \mathrm{(1-octanethiol),} \mathrm{biotin,} \mathrm{anti-}$ biotin or streptavidin (Fig. 8) [41].

Moreover, DNA conjugation combined with UV-visible spectroscopy and SPR spectroscopy can be used for observing the protein conformational changes. There is a color shift in the solution of the yeast iso-1-cytochrome (Cyt c) protein covering a gold nanoparticle by the conformational change in the protein which can be observed by UV-visible absorption spectroscopy. In addition, refractive index of the flat gold films covered by the same protein changes again by conformational change of the protein. This change in refractive index is monitored by surface plasmon resonance spectroscopy [13] (Figs 9 and 10).

Streptavidin-biotin is a good choice to combine with quantum dots in sensor applications, because the streptavidin-biotin complex has an excellent binding ability (Fig. 8) [37,41].

Furthermore, there are several colorimetric biosensor applications with the use of UV-visible spectroscopy combined with DNA conjugation of metal NCs which can detect various analytes including ions or organic molecules by observing the changes in the heights or shifts of the peaks. If the colorimetric detection of $\mathrm{Pb}^{+2}$ is considered as an example, it is observed that the color of the solution of DNAzyme (Catalytically active DNA) conjugated gold nanoparticles is changed from blue to purple to red as the $\mathrm{Pb}^{+2}$ concentration is increased from 0 to $5 \mu \mathrm{m}[20]$. 
(A) GroEL
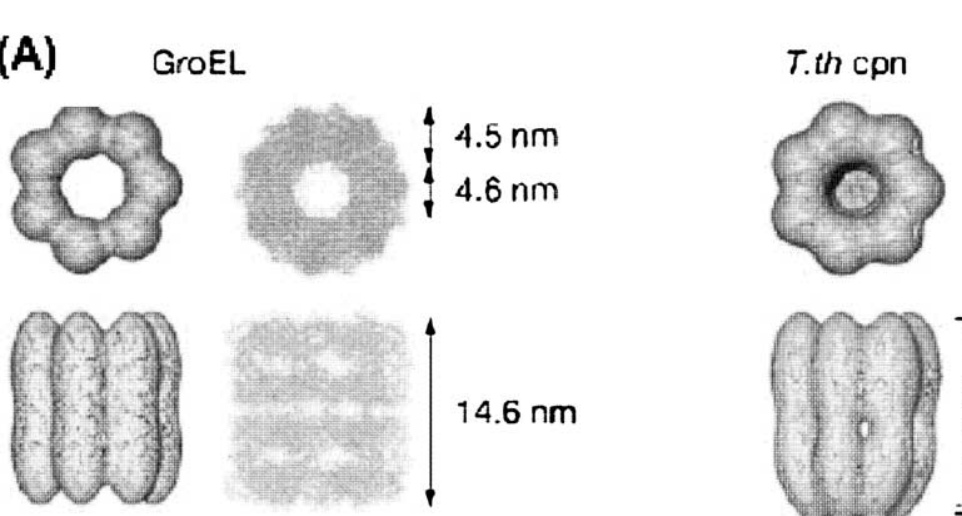

$14.6 \mathrm{~nm}$

Crystal structure

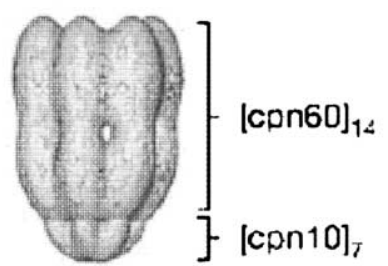

(B)|

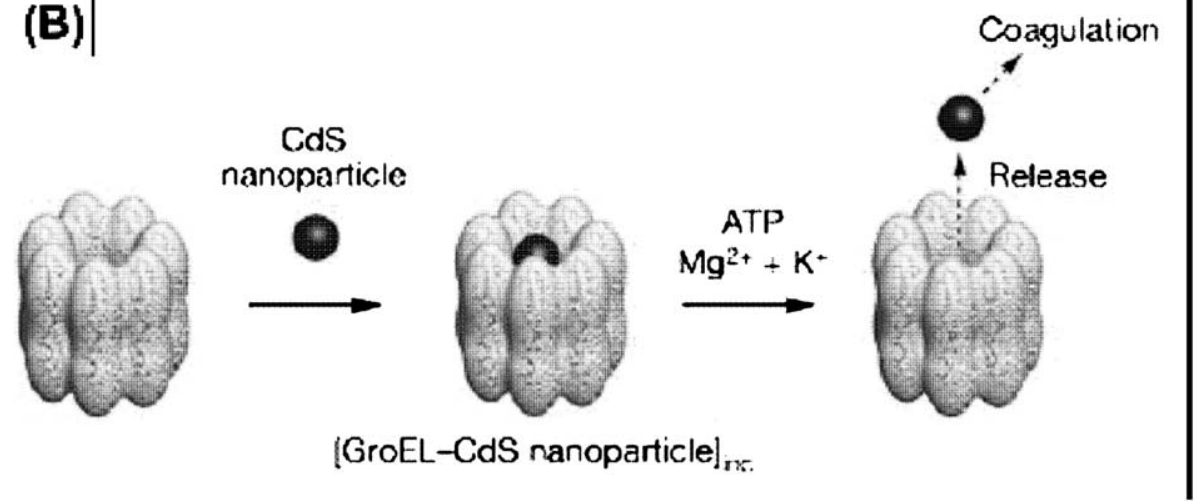

Fig. 6. Chaperonin proteins as ATP-responsive barrels for inclusion of nanoparticles. (A) Schematic illustrations (top and side views) of GroEL (crystal structures, right) and T.th cpn. (B) Schematic representation of the formation of GroEL-CdS nanoparticle complexes by inclusion of CdS nanoparticles into the cylindrical cavity of GroEL, and its ATP-triggered guest release. Reproduced with permission from Nature 423 (2004), 628-632.

In addition to all, the problems of near field detection such as background fluorescence and low signal magnitude in Raman spectroscopy can be overcome by treating biomaterials with silver nanoparticles [42].

Finally, there are studies of in vivo imaging of biological materials using quantum dots $[9,30,35$, 40,43]. However, limited penetration depth of visible light does not allow deep tissue imaging [40]. Moreover, the problem of cell auto fluorescence in the visible range prevents clear observations of the fluorescence from the labels. This situation is overcome by the use of QDs emitting in infrared (IR) region [44]. By several spectroscopic and microscopic studies these particles can be tracked in biological tissue [40,44]. The photoproperties of $752 \mathrm{~nm}$ near infrared QDs are shown in Fig. 11 [45].

\section{Conclusion}

The discovery of nanocrystals and their use in biological sciences have opened new windows to the researchers. Their sharp and narrow emission peaks, and ability to suffer less from photobleaching and to be excited by a single source in addition to the ability of conjugating them with chemical and biological 

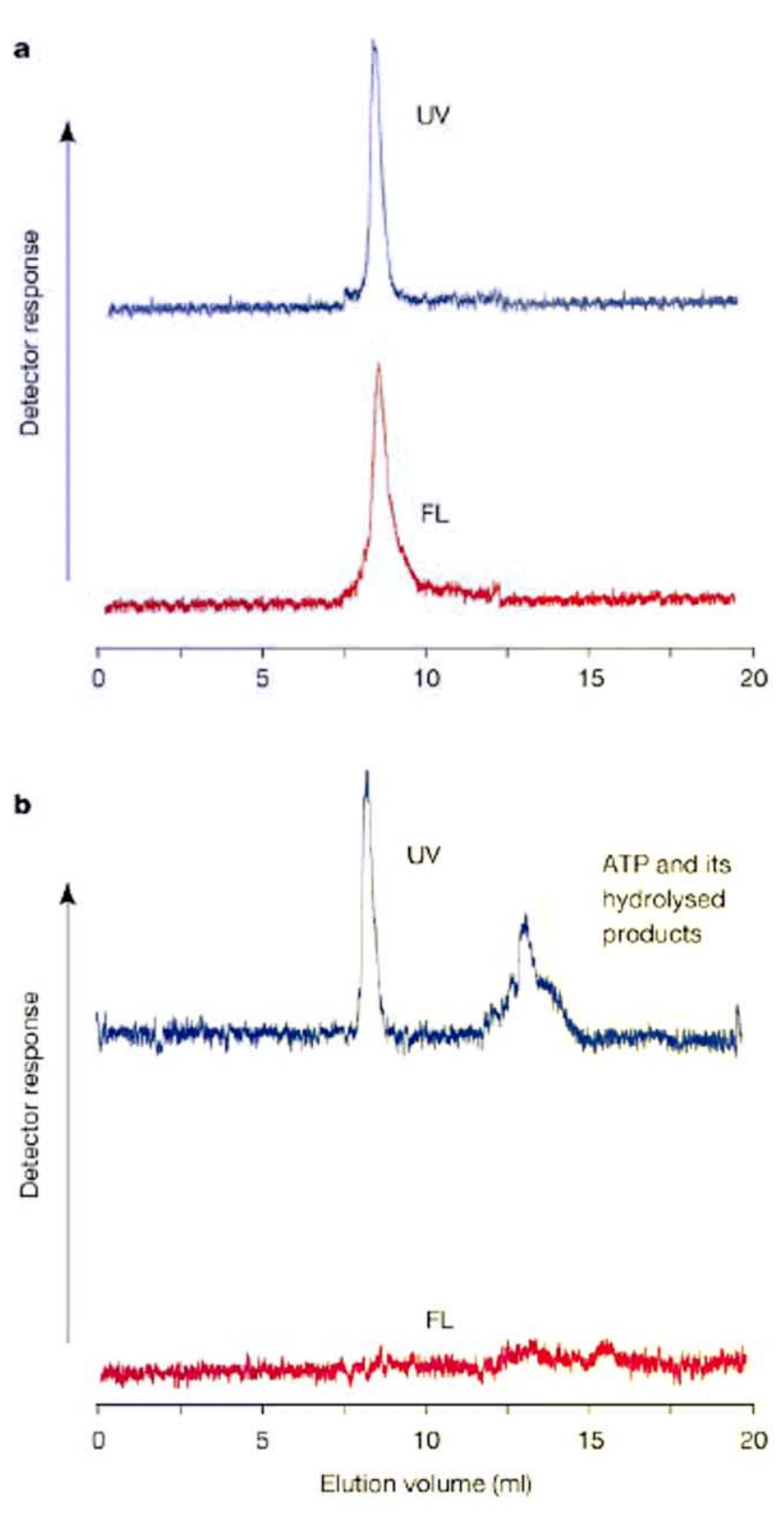

Fig. 7. SEC analysis of T.th cpn-CdS the nanoparticle inclusion complex and its specific response to ATP in KCl-containing Tris- $\mathrm{HCl}$ buffer. (A) Analytical SEC trace of T.th cpn-CdS inclusion complexes monitored by an UV (upper chart)/fluorescence (FL) (lower chart) dual detector, and (B) that of T.th cpn-CdS inclusion complexes after addition of ATP and $\mathrm{MgCl}_{2}$. The peak at $12.5-15.0 \mathrm{ml}$ is derived from ATP and its hydrolysed products. Reproduced with permission from Nature $\mathbf{4 2 3}$ (2004), $628-632$. 
(A)|

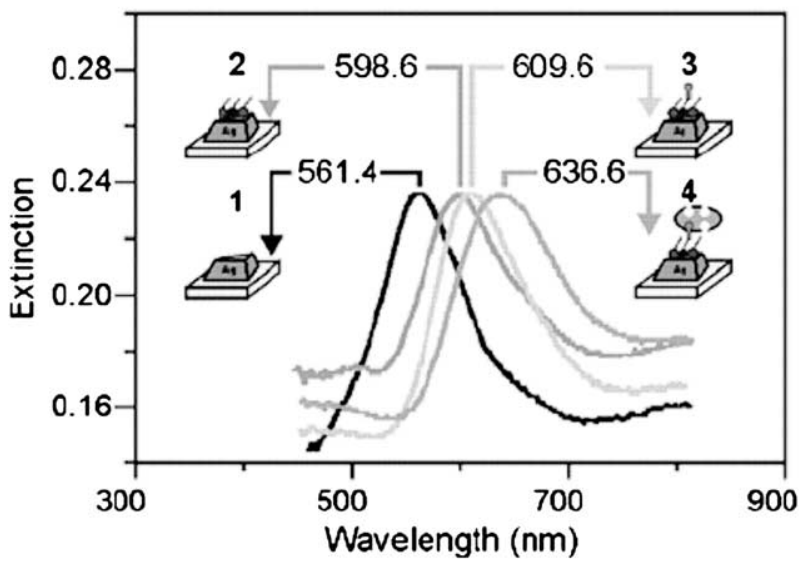

(B)

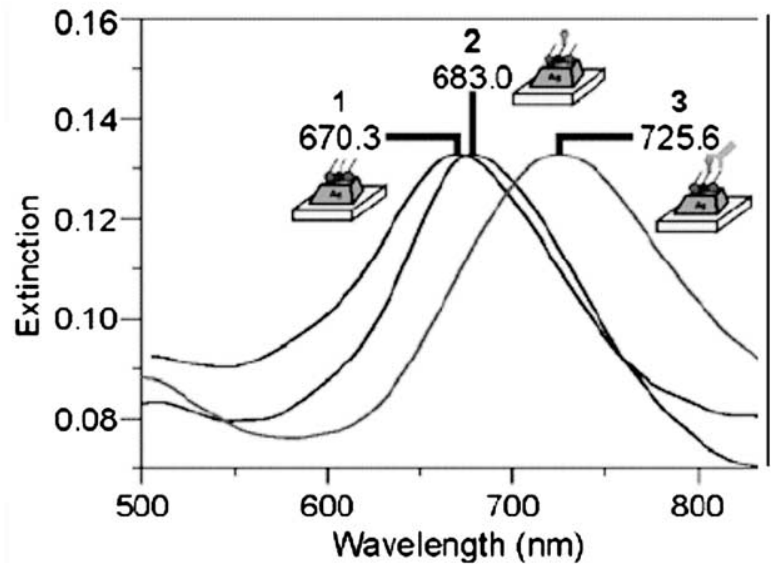

Fig. 8. (A) LSPR spectra of each step in the surface modification of NSL-derived Ag nanocparticles to form a biotinylated Ag biosensor and the specific binding streptavidin. (1) Ag nanoparticles before chemical modification, $\lambda_{\max }=561.4 \mathrm{~nm}$. (2) Ag nanoparticles after modification with $1 \mathrm{mM} \mathrm{1:3} \mathrm{11-MUA} \mathrm{(11-mercaptoundecanoic} \mathrm{acid):} \mathrm{1-OT} \mathrm{(1-octanethil),}$ $\lambda_{\max }=598.6 \mathrm{~nm}$. (3) Ag nanoparticles after modification with $1 \mathrm{mM}$ biotin, $\lambda_{\max }=609.6 \mathrm{~nm}$. (4) $\mathrm{Ag}$ nanoparticles after modification with $100 \mathrm{nM}$ streptavidin, $\lambda_{\max }=636.6 \mathrm{~nm}$. All extinction measurements were collected in a $\mathrm{N}_{2}$ environment. (B) Smoothed LSPR spectra for each step of the preparation of the Ag nanobiosensor, and the specific binding of anti-biotin to biotin. (1) Ag nanoparticles after modification with $1 \mathrm{mM} \mathrm{3:11-OT/11-MUA,} \lambda_{\max }=670.3 \mathrm{~nm}$, (2) Ag nanoparticles after modification with $1 \mathrm{mM}$ biotin, $\lambda_{\max }=683.0 \mathrm{~nm}$. And (3) Ag nanoparticles after modification with $700 \mathrm{nM}$ anti-biotin, $\lambda_{\max }=725.6 \mathrm{~nm}$. All spectra were collected in a $\mathrm{N}_{2}$ environment. Reproduced with permission from SPIE 5221 (2003), $47-58$.
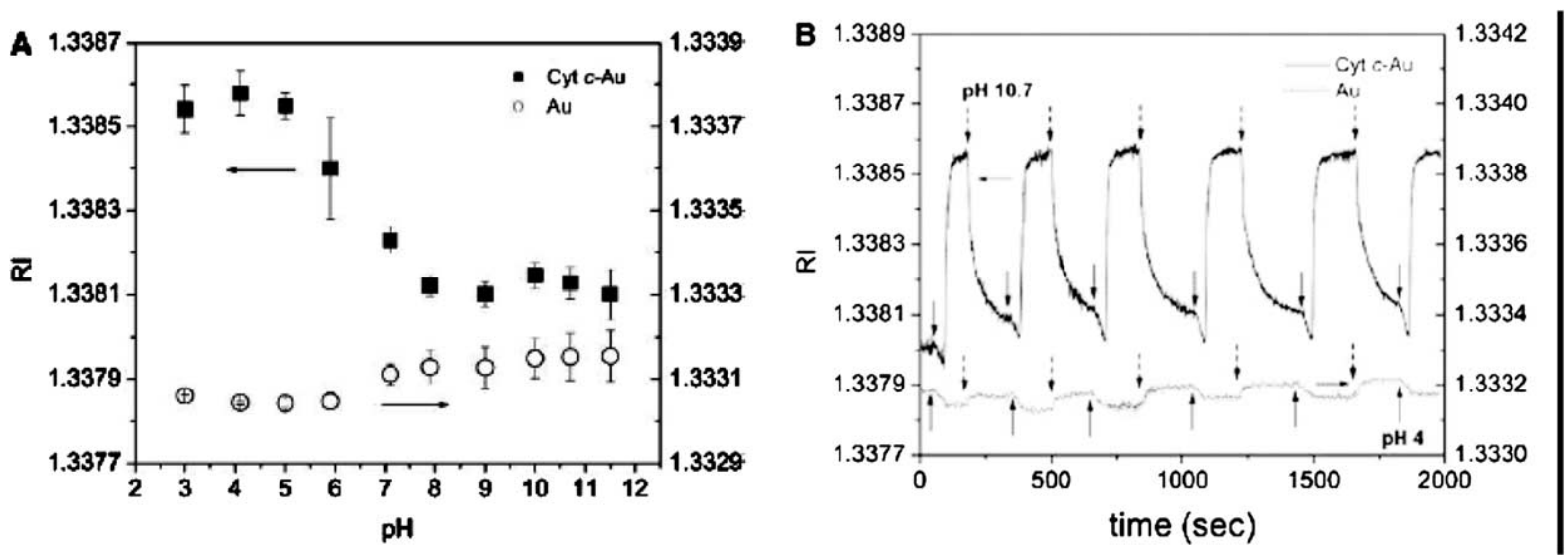

Fig. 9. SPR data for both bare Au and Cyt c-Au (A) Refractive index (RI) measured on both bare Au (open circles) and Cyt c-Au (solid squares). The RI for bare Au increases while that for Cyt c-Au decreases. The arrows point to the vertical axis associated with each data set. (B) Reversibility and reproducibility of the SPR signal of Cyt c bound to Au. The conformational change of $\mathrm{Cyt} \mathrm{c}$ on $\mathrm{Au}$ was reversible and reproducible when the $\mathrm{pH}$ of the solution in contact with the gold film was repeatedly varied from $\mathrm{pH} 4$ to $\mathrm{pH}$ 10.7. Solid arrows and the dotted arrows represent injection times of $\mathrm{pH} 4$ and $\mathrm{pH} 10.7$ solutions, respectively. The vertical axis on the left is for Cyt c bound to Au whereas the vertical axis on the right is for bare Au. Reproduced with permission from Chem. Biol. 12 (2005), 323-328. 


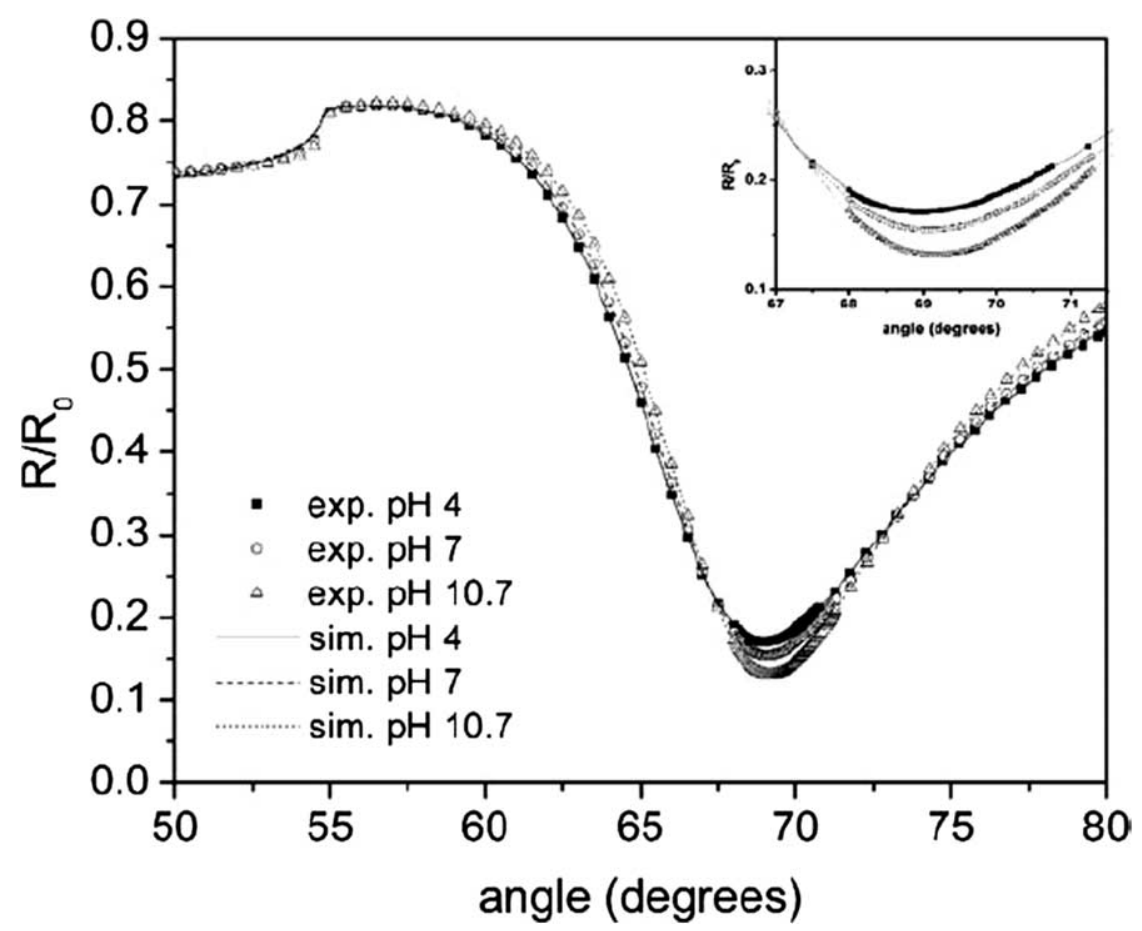

Fig. 10. Experimental angle resolved SPR signals for three different $\mathrm{pH}$ conditions solid squares, open circles, and open triangles are for $\mathrm{pH} 4, \mathrm{pH} 7$, and $\mathrm{pH}$ 10.7, respectively. The best fitting simulation data for obtaining dielectric constants and thickness of the Cyt c layer is represented by a solid line for $\mathrm{pH} 4$, a dashed line for $\mathrm{pH} 7$, and a dotted line for $\mathrm{pH}$ 10.7. The inset shows a magnified portion of this figure over the range from $67^{\circ} \mathrm{C}$ to $71.5^{\circ} \mathrm{C}$. Reproduced with permission from Chem. Biol. 12 (2005), $323-328$.

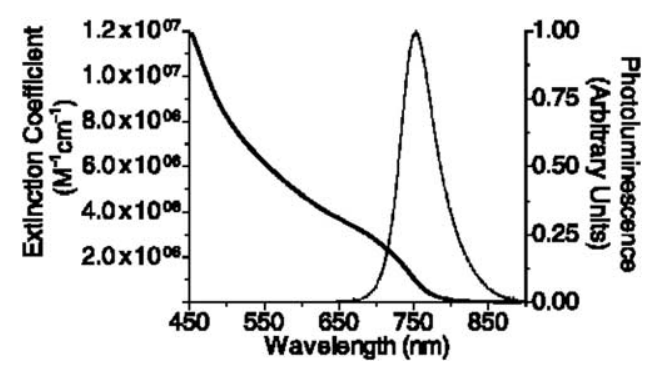

Fig. 11. Photoproperties of $752 \mathrm{~nm}$ NIR QDs. The core of the above QD is CdTe, and the shell is CdSe. Extinction coefficient is shown on the left axis (thick solid red line) and the photoluminescence (500 nm excitation) is shown on the right axis (dashed line), both as a function of wavelength. Reproduced with permission form Mol. Imag. 2 (2003), 50-64.

materials gave researchers talented tools in biological spectroscopy and microscopy. Nanocrystals are not only used for detection: Conjugation of nanocrystals with the biological molecules are opened new windows to the nanoscience by allowing them to be used as building blocks for larger nano- and microstructures. It is clear that the intense studies worldwide will provide new explorations about the synthesis and utilization of nanocrystals. 


\section{References}

[1] W.J. Parak, D. Gerion, T. Pellegrino, D. Zanchet, C. Micheel, S.C. Williams, R. Boudreau, M.A. Le Gros, C.A. Larabell and A.P. Alivisatos, Nanotechnology 14 (2003), 15-17.

[2] S. Fafard, H.C. Liu, Z.R. Wasilewski, J. McCaffrey, M. Spanner, S. Raymond, C. N. Allen, K. Hinzer, J. Lapointe, C. Struby, M. Gao, P. Hawrylak, C. Gould, A. Sacrajda and P. Zawadzki, SPIE 4078 (2000), 100-114.

[3] P. Hawrylak, S. Fafard and Z. Wasilewski, Cond. Matt. News 7 (1999), 16-25.

[4] J. Oullette, The Industrial Physicist 9 (2003), 14-17.

[5] D.L. Klein, R. Roth, A.K.L. Lim, A.P. Alivisatos and P.L. McEuen, Nature 389 (1997), 699-701.

[6] H.C. Liu, B. Aslan, M. Korkusinski, S.J. Cheng and P. Hawrylak, Infrared Physics \& Technology 44 (2003), 503-508.

[7] D. Ishii, K. Kinbara, Y. Ishida, N. Ishii, M. Okochi, M. Yohda and T. Aida, Nature 423 (2003), 628-632.

[8] A.J. Haes, D.A. Stuart, S. Nie and R.P. Van Duyne, Journal of Fluorescence 14 (2004), 355-367.

[9] M.E. Akerman, W.C.W. Chan, P. Laakkonen, S.N. Bhatia and E. Ruoslahti, Proc. Nat. Acad. Sci. 99 (2002), 12617-12621.

[10] D.R. Larson, W.R. Zipfel, R.M. Williams, S.W. Clark, M.P. Bruchez, F.W. Wise and W.W. Webb, Science 300 (2003), 1434-1436.

[11] M. Ozkan, DDT 9 (2004), 1065-1071.

[12] W.C.W. Chan, D.J. Maxwell, X. Gao, R.E. Bailey, M. Han and S. Nie, Current Opinion in Biotechnology 13 (2002), 40-46.

[13] S. Chah, M.R. Hammond and R.N. Zare, Chem. Biol. 12 (2005), 323-328.

[14] E. Braun, Y. Eichen, U. Sivan and G. Ben-Yoseph, Nature 391 (1998), 775-778.

[15] J. Richter, M. Mertig, W. Pompe, I. Mönch and H.K. Schackert, Appl. Phys. Lett. 78 (2001), 536-538.

[16] E. Winfree, F. Liu, L.A. Wenzler and N.C. Seeman, Nature 394 (1998), 539-544.

[17] D. Gerion, W.J. Parak, S.C. Williams, D. Zanchet, C.M. Micheel and A.P. Alivisatos, J. Am. Chem. Soc. 124 (2002), 7070-7074.

[18] N.C. Seeman, Annu. Rev. Biophys. Biomol. Struct. 27 (1998), 225-248.

[19] C.P. Collier, T. Vossmeyer and J.R. Heath, Annu. Rev. Phys. Chem. 49 (1998), 371-404.

[20] J. Liu and Y. Lu, J. Am. Chem. Soc. 125 (2003), 6642-6643.

[21] A. Kumar, S. Phadtare, R. Pasricha, P. Guga, K.N. Ganesh and M. Sastry, Curr. Sci. 84 (2003), 71-74.

[22] P. Alivisatos, Pure Appl. Chem. 72 (2000), 3-9.

[23] M. Dahan, S. Lévi, C. Luccardini, P. Rostaing, B. Riveau and A. Triller, Science 302 (2003), 442-445.

[24] Z. Kaul, T. Yaguchi, S.C. Kaul, T. Hirano, R. Wadhwa and K. Taira, Cell Res. 13 (2003), 503-507.

[25] X. Michalet, F. Pinaud, T.D. Lacoste, M. Dahan, M.P. Bruchez, A.P. Alivisatos and S. Weiss, Single Mol. 2 (2001), 261276.

[26] S.M. Sze, Modern Semiconductor Physics, John Wiley \& Sons Inc, New York, 1998.

[27] D. Bimberg, M. Grundmann and N.N. Ledentsov, Quantum Dot Heterostructures, John Wiley \& Sons Inc, New York, 1998.

[28] M.A. Reed, Scientific American 298 (1993), 118-123.

[29] L. Jacak, P. Hawrylak and A. Wojs, Quantum Dots, Springer, Berlin, 1998.

[30] X. Michalet, F.F. Pinaud, L.A. Bentolila, J.M. Tsay, S. Doose, J.J. Li, G. Sundaresan, A.M. Wu, S.S. Gambhir and S. Weiss, Science 307 (2005), 538-544.

[31] A. Chrowos, I. Severcan, A.Y. Koyfman, P. Weinkam, E. Oroudjev, H.G. Hansma and L. Jaeger, Science 306 (2004), 2068-2072.

[32] C.M. Niemeyer, Angew. Chem. Int. Ed. Engl. 40 (2001), 4128-4158.

[33] Y. Eichen, E. Braun, U. Sivan, G. Ben-Yoseph, Acta Polym. 49 (1998), 663-670.

[34] N.C. Seeman and A.M. Belcher, Proc. Nat. Acad. Sci. 99 (2002), 6451-6455.

[35] B. Dubertret, P. Skourides, D.J. Norris, V. Noireaux, A.H. Brivanlou and A. Libchaber, Science 298 (2002), $1759-1762$.

[36] E.R. Goldman, E.D. Balighian, H. Mattoussi, M.K. Kuno, J.M. Mauro, P.T. Tran and G.P. Anderson, J. Am. Chem. Soc. 124 (2002), 6378-6382.

[37] A.J. Haes, D.A. Stuart, S. Nie and R.P. Van Duyne, Journal of Fluorescence 14 (2004), 355-367.

[38] W.C.W. Chan and S. Nie, Science 281 (1998), 2016-2018.

[39] H.E. Grecco, K.A. Lidke, R. Heintzmann, D.S. Lidke, C. Spagnuolo, O.E. Martinez, E.A. Jares-Erijman and T.M. Jovin, Mic. Res. Tech. 65 (2004), 169-179.

[40] X. Gao, L. Yang, J.A. Petros, F.F. Marshall, J.W. Simons and S. Nie, Current Opinion in Biotechnology 16 (2005), 63-72.

[41] A.J. Haes and R.P. Van Duyne, SPIE 5221 (2003), 47-58.

[42] S.R. Emory and S. Nie, Anal. Chem. 69 (1997), 2631-2635.

[43] O.V. Salata, J. Nanobiotechnology 2 (2004), 12.

[44] E. Sargent, Adv. Mater. 17 (2005), 515-522.

[45] Y.T. Lim, S. Kim, A. Nakayama, N.E. Stott, M.G. Bawendi and J.V. Frangioni, Mol. Imag. 2 (2003), 50-64. 


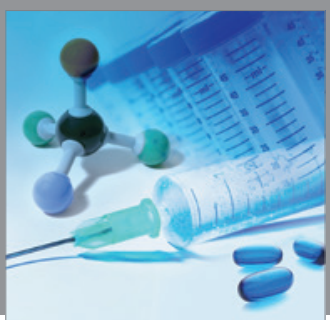

International Journal of

Medicinal Chemistry

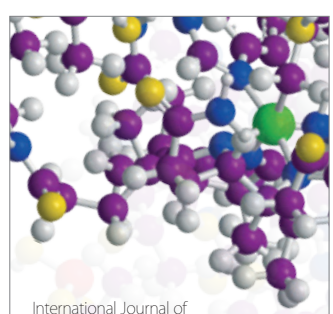

Carbohydrate Chemistry

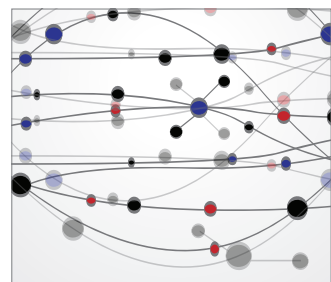

The Scientific World Journal
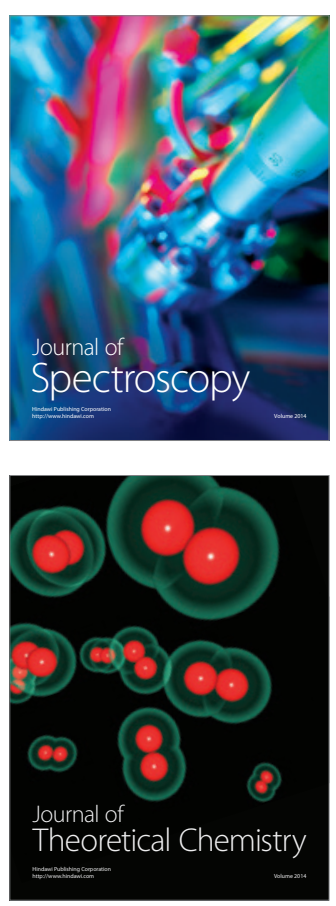
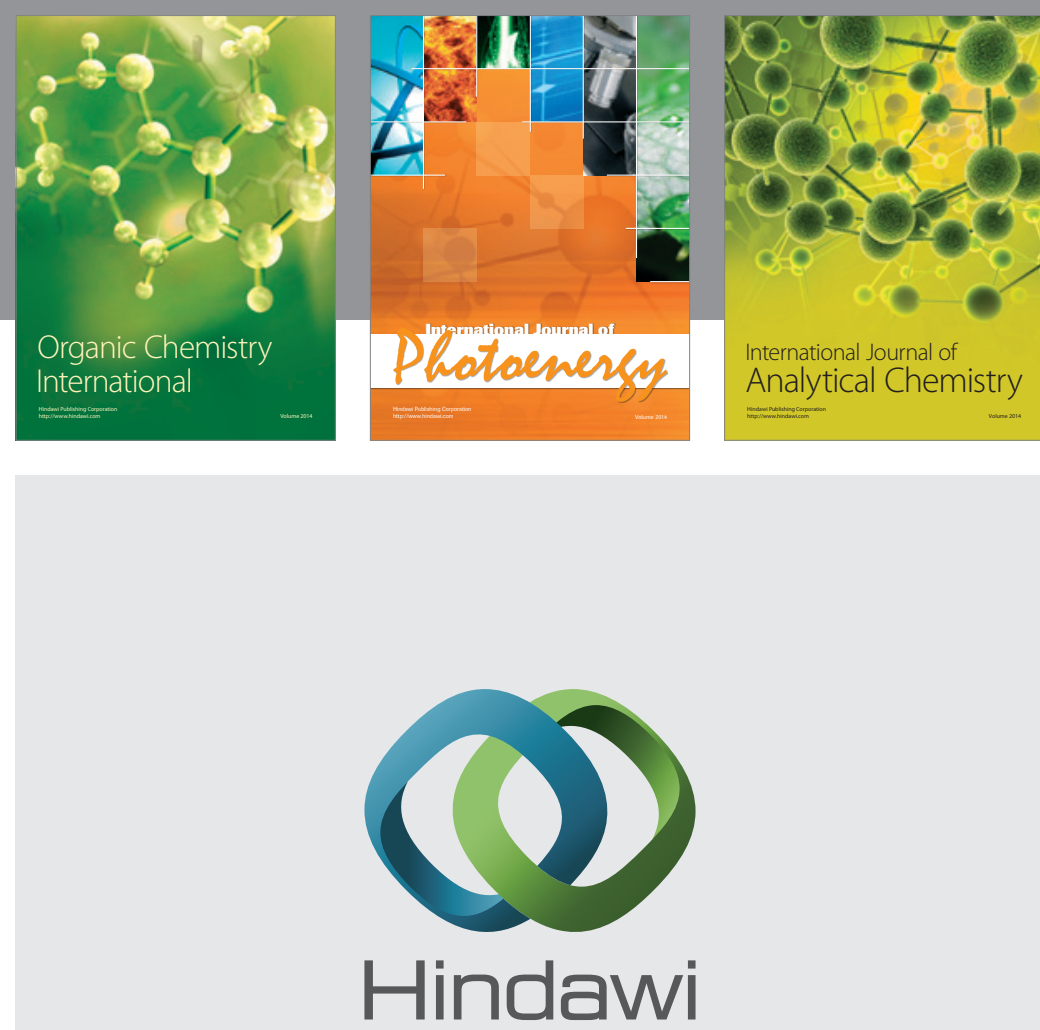

Submit your manuscripts at

http://www.hindawi.com
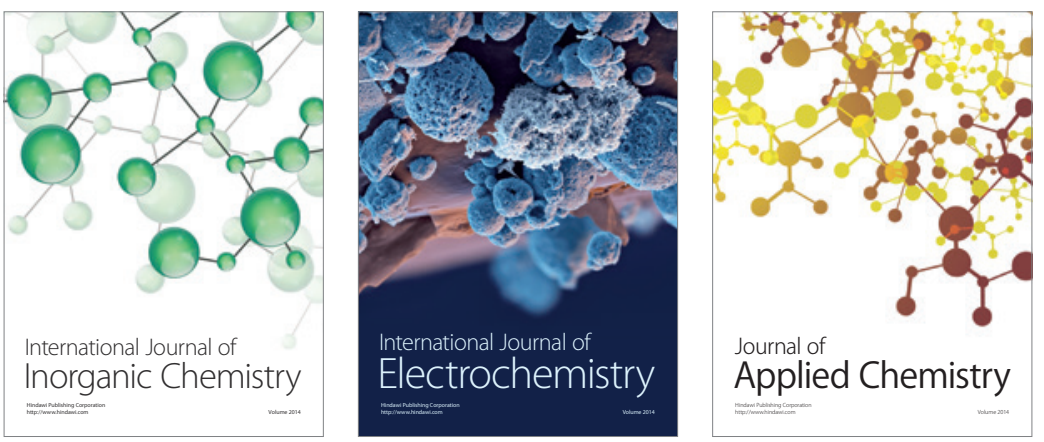

Journal of

Applied Chemistry
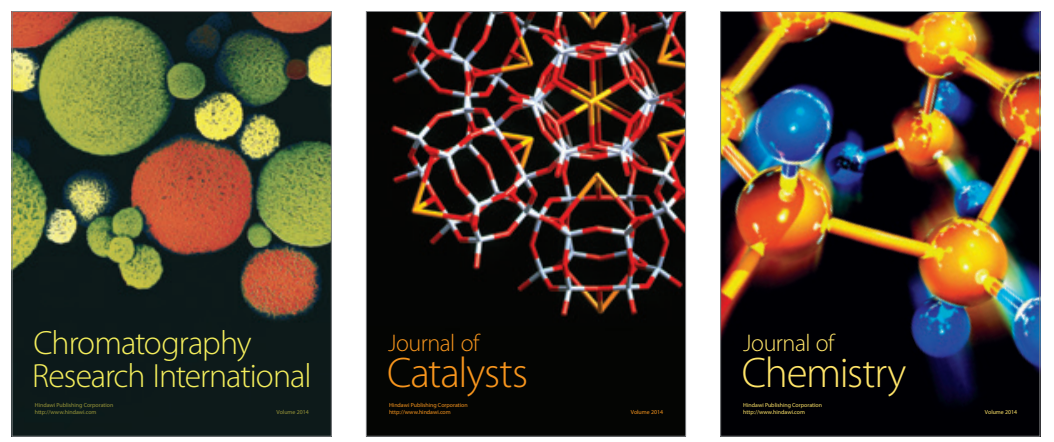
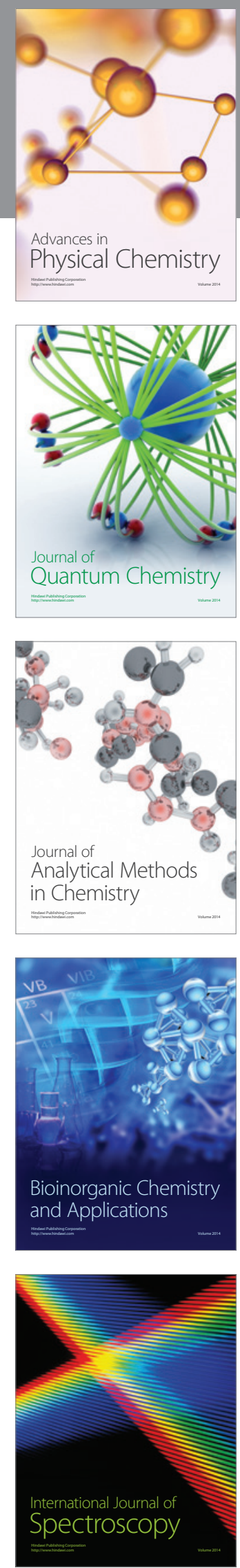